\title{
Avoid hypotension and hypoxia: an old anesthetic adage with renewed relevance from cerebral oximetry monitoring
}

\author{
Hilary P. Grocott, MD
}

Published online: 4 June 2011

(C) Canadian Anesthesiologists' Society 2011

In this freestanding editorial, issues are discussed regarding advancements in patient monitoring modalities and how they are poised to continue the evolution of perioperative management. Innovations in cerebral oximetry technologies using near infrared spectroscopy (NIRS) is utilized may allow us to reconsider how we define and manage both hypotension and hypoxemia. With the exception of cardiac anesthesia where cerebral oximetry had early (though arguably still sporadic) clinical adoption, its dispersion into other perioperative disciplines has been somewhat limited. However, the information this technology provides could significantly alter how we manage many physiologic perturbations seen during anesthesia. As a corollary to the scrutiny directed at pulse oximetry when it first came into clinical practice, cerebral oximetry is on the precipice of an increasingly steeper adoption curve which demands a similar evaluation of its uses and potential efficacy. The purpose of this editorial is to discuss how the use of NIRS cerebral oximetry may require clinicians to re-examine their understanding of perioperative physiologic management, particularly that related to blood pressure and oxygenation.

In days gone by, the phrase, "avoid hypotension and hypoxia", was a remark appearing frequently in the preoperative medical consultations requested by surgeons for seemingly higher-risk patients. This cautionary statement seemed so obvious that many anesthesiologists caring for these patients viewed it as downright insulting. Indeed, the consequent seething criticism of our medical colleagues

H. P. Grocott, MD ( $₫)$

Departments of Anesthesia and Surgery, University of Manitoba, CR3008-369 Tache Avenue, Winnipeg, MB R2H 2A6, Canada e-mail: hgrocott@sbgh.mb.ca was frequent fodder for breakroom chatter. No one could argue that this suggestion was in any way erroneous, yet avoiding these two clinical situations was seen as so obvious and basic a tenet that even the most junior anesthetic trainee could be counted on to avoid these situations - or, at the very least, to identify them and take counteractive measures.

However, in the ensuing decades, a time during which technologic advances have reassured us - perhaps falsely that we are detecting these occurrences accurately and preventing them as much as possible, it is increasingly becoming apparent that our confidence in doing so may be misplaced. Indeed, to avoid these situations assumes our competence both to detect them and to intervene accordingly. However, detection is, in part, critically dependent upon how hypotension and hypoxia are defined. For example, hypotension should be determined according to the patient's baseline blood pressure status and, as such, should be individualized. Instead, it is placed too often in the context of the somewhat arbitrarily defined normal limits of autoregulation-dependent tissue perfusion.

The cerebral autoregulatory curve outlines the relationship between blood pressure and cerebral blood flow $(\mathrm{CBF})$. The pressure independent central plateau (though even under normal conditions it has a slightly positive slope $^{1}$ ) is bordered by pressure dependent steeply sloped regions at both low and high pressure thresholds (i.e., knees of the curve). A number of pathologic conditions and perioperative situations encountered relatively routinely (e.g., hypercarbia or volatile anesthesia levels $>1$ MAC) can alter the slope and/or the knees of the plateau. Although the lower threshold for cerebral autoregulation has traditionally been defined as approximately $50 \mathrm{mmHg}$, this is variable and may be influenced in particular by the presence of pre-existing hypertension. ${ }^{2}$ Even in the absence 
of hypertension, interpatient heterogeneity in autoregulation (particularly at the lower threshold) possibly superimposed on variable degrees of vascular occlusive disease questions the validity of arbitrary definitions of normal blood pressure and makes it difficult to define hypotension with any unifying number.

Cerebral oximetry, a noninvasive optical technology employing NIRS, may allow for real-time determination of the various autoregulatory thresholds ${ }^{3}$ and thereby provide relevance as a means to define clinically meaningful hypotension. Cerebral oximetry measures regional cerebral blood (representing approximately $75 \%$ venous and $25 \%$ arterial content) oxygen saturation $\left(\mathrm{rSO}_{2}\right)$ using principles similar to conventional pulse oximetry (i.e., Beer-Lambert Law) which allow for the determination of oxygenated and deoxygenated hemoglobin by the differential absorption of various near-infrared light wavelengths. ${ }^{4}$ Reductions in $\mathrm{rSO}_{2}$ reflect an increased oxygen extraction ratio which can result from reduced oxygen delivery (the usual culprit) or increased utilization. A number of physiologic variables can effect a change in $\mathrm{rSO}_{2}{ }^{5}$ For example, the dynamic response of $\mathrm{rSO}_{2}$ to changes in $\mathrm{PaCO}_{2}$ is well known with even mild degrees of hypocapnia (i.e., $\mathrm{PaCO}_{2}<35 \mathrm{mmHg}$, which is common and often overlooked during routine anesthetic care) resulting in significant decreases in CBF and oxygen delivery with corresponding reductions in $\mathrm{rSO}_{2}$.

Cerebral oximetry may soon allow for the optimization of perioperative blood pressure and provide information to define when low is too low. However, when considering the implications of intraoperative hypotension, the pressure threshold (i.e., the severity of hypotension) must be defined together with the duration of time below this threshold; integrating these concepts presents the area under the curve (AUC) as an overall index of hypotension exposure. This approach addresses the issue as to whether brief but significant intraoperative hypotension (e.g., as seen at anesthesia induction) is as crucial as more prolonged though perhaps less severe (in terms of absolute blood pressure levels) periods of hypotension. Once more, a NIRS cerebral oximetry corollary has also been demonstrated to reflect the impact of hypotension on outcomes. Indeed, in a study of patients undergoing aortic arch surgery, Fischer et al. demonstrated a significant relationship between adverse outcomes and both AUC and time under the curve for several $\mathrm{rSO}_{2}$ thresholds. ${ }^{6}$ In noncardiac surgery, the impact of relatively mild though prolonged periods of hypotension (often underrecognized due to issues related to blood pressure cuff placement in patients in the sitting position) is now being addressed using cerebral oximetry. Evidence from orthopedic patients under going shoulder surgery points to the utility of NIRS in detecting less severe but potentially prolonged hypotension and its effects on outcome. ${ }^{7}$
Using cerebral tissue oxygen saturation as an indirect measure of relative CBF could facilitate outlining the autoregulatory relationship between blood pressure and CBF. Indeed, evidence linking the impairment in NIRS-derived autoregulation and adverse neurologic outcomes in cardiac surgery suggests the potential for real-time monitoring that may identify opportunities to alter outcome. ${ }^{8}$ As a result, the determination of optimal pressure for each individual can replace the more generalized approach to blood pressure inherent in the population heterogeneity. Despite an ever growing body of literature which is repeatedly describing an association between impaired $\mathrm{rSO}_{2}$ and adverse perioperative outcomes, ${ }^{6,9-12}$ the potential benefits of an intuitive approach to the individualization of care, although showing great promise, awaits testing in clinical trials that are properly designed and executed.

Just as defining and treating hypotension may need reconsideration and clinical context, hypoxia, which appears, at first glance, as an obvious clinical occurrence to define, also warrants a second look with reference to cerebral oximetry. Hypoxemia, defined by a low partial pressure of oxygen in the blood, results in lower than normal levels of oxygen in tissues. However, there clearly are conditions whereby the arterial blood oxygen content is sufficiently high not to meet any obvious threshold for hypoxemia, yet there are reduced levels of oxygen at the tissue level than are needed for aerobic metabolism. Paradoxically, there is also evidence suggesting a "normal" oxygen saturation is not ideal for some. Across the spectrum of human age, tolerance for relative hypoxia varies. The very young, such as premature neonates, may have a tolerance, if only transient (i.e., in the early days of life), for a lower oxygen content, and they may be harmed by too much oxygen. Similarly, pediatric patients with cyanotic congenital heart disease may be harmed by otherwise normal oxygen saturation.

Cerebral oximetry can provide information regarding oxygen delivery and utilization that now allows insight into whether a patient may be exhibiting tissue hypoxia despite normal systemic arterial saturation and adequate blood pressure. Ensuring maintenance of optimal tissue (particularly brain) oxygen saturation requires real-time assessment of the multiple determinants of tissue oxygenation, including factors related to oxygen delivery (i.e., pressure, blood flow, and oxygen content) and utilization. Until recently, understanding how to integrate these individual variables has been flawed and dependent upon general assumptions. Near infrared spectroscopy cerebral oximetry allows the integration of oxygen content (determined by hemoglobin and $\mathrm{PaO}_{2}$ ), blood flow, and pressure and permits insight into tissue metabolism. Thus, individualized care can be provided to patients who present for perioperative care with increasingly complex conditions. 
The eventual targets for defining relevant hypotension and hypoxia (i.e., the diagnostic utility of NIRS) must be placed in the context of how therapy will be guided (i.e., the therapeutic utility of NIRS). These roles are inextricably linked, with therapeutic intervention guided by these diagnostic technologies. However, tempering any enthusiasm as to how NIRS may improve outcomes is the issue of linking monitoring strategy to improved outcome. Indeed, such enthusiasm for a generalized benefit to the SwanGanz catheter, for example, was eventually debunked by research in the decades that followed its adoption. ${ }^{13}$ It has not been demonstrated that pulse oximetry, a standard of care in our practice, improves outcome, ${ }^{14}$ though it clearly has an intuitive benefit. Furthermore, the changes in outcome resulting from interventional therapy (albeit guided by the monitor itself) confound any potential benefit from an individual monitor.

The evolving complexities of perioperative physiologic monitoring (and related therapeutic options) that have surfaced in light of expanding NIRS technologies suggest that the once static understanding of hypotension and hypoxia clearly needs reconsideration. Cerebral oximetry exemplifies these issues and will no doubt serve as a focus of debate regarding the ifs and hows to optimize outcomes with its use. That said, there appears to be sufficient renewed relevance to the old anesthetic adage of avoiding hypotension and hypoxia, and we must remain openminded in our approach to the principle.

\section{Éviter l'hypotension et l'hypoxie: un vieil adage en anesthésie dont la pertinence est renouvelée à cause du monitorage de l'oxymétrie cérébrale}

Dans cet éditorial indépendant, nous aborderons certaines questions liées à l'influence des percées au niveau des modalités de monitorage des patients sur l'évolution de la prise en charge périopératoire. Les innovations technologiques dans la spectroscopie proche infrarouge (NIRS), une modalité de plus en plus accessible sous forme d'oxymétrie cérébrale par exemple, pourraient nous permettre de repenser notre définition et notre prise en charge de l'hypotension et de l'hypoxémie. Hormis en anesthésie cardiaque, domaine dans lequel l'oxymétrie cérébrale a été rapidement adoptée pour des applications cliniques (bien que cette adoption soit toujours quelque peu éparse), cette modalité n'a que peu pénétré les autres disciplines périopératoires. Les renseignements qu'elle fournit pourraient pourtant modifier considérablement la prise en charge de nombre des perturbations physiologiques observées pendant l'anesthésie. Tout comme l'oxymétrie de pouls, qui fut soumise à un examen minutieux lors de son apparition en pratique clinique, l'oxymétrie cérébrale, qui elle aussi est adoptée de plus en plus rapidement, doit faire l'objet d'une évaluation semblable de ses utilisations et de son efficacité potentielles. L'objectif de cet éditorial est d'estimer comment l'utilisation de l'oxymétrie cérébrale à NIRS pourrait pousser les cliniciens à réévaluer leur compréhension de la prise en charge physiologique périopératoire, particulièrement en ce qui touche à la tension artérielle et à l'oxygénation.

Jadis, la mention « éviter l'hypotension et l'hypoxie » figurait souvent dans les consultations médicales périopératoires demandées par les chirurgiens pour les patients présentant apparemment un risque de complications plus élevé que la normale. Cette recommandation était tellement évidente qu'elle était perçue comme insultante par nombre d'anesthésiologistes prenant soin de tels patients. En effet, les critiques virulentes qui s'ensuivaient à l'endroit de nos collègues médicaux a animé plus d'un bavardage en salle de repos. Personne ne dira que cette suggestion est erronée de quelque façon que ce soit; plutôt, le fait d'éviter ces deux situations cliniques était considéré comme tellement évident et fondamental que même le résident en anesthésie le plus inexpérimenté savait éviter ces situations - ou du moins les reconnaître et prendre les mesures nécessaires pour les contrer.

Pourtant, au cours des décennies suivantes, les progrès technologiques nous ont rassuré - peut-être de façon erronée - en nous rendant confiants que nous dépistons de façon précise ces situations et les prévenons autant que possible; il devient cependant de plus en plus évident que notre confiance pourrait avoir été mal placée. En effet, il faut prendre pour acquis que, pour éviter de telles situations, nous les dépistons systématiquement et que nous intervenons en conséquence. Le dépistage dépend toutefois de façon critique - en partie du moins - de notre définition de l'hypotension et de l'hypoxie. Par exemple, l'hypotension doit tenir compte de la tension artérielle de base du patient et doit, dès lors, être personnalisée. Au lieu de cela, elle est souvent évaluée par rapport aux limites normales de l'autorégulation pour la perfusion tissulaire, lesquelles sont définies plus ou moins arbitrairement.

La courbe d'autorégulation cérébrale décrit la relation entre la tension artérielle et le débit sanguin cérébral (DSC). Le plateau central où le débit est indépendant de la tension (bien qu'il possède, même en conditions normales, une pente légèrement positive) ${ }^{1}$ est entouré, au-delà des seuils de tension basse et élevée (ou coudes de la courbe), de régions à pente prononcée, où le débit dépend de la tension. Plusieurs conditions pathologiques, ainsi que 
certaines situations périopératoires relativement courantes (telles que l'hypercarbie ou des niveaux d'anesthésiques volatils en concentration alvéolaire maximale (CAM) supérieure à 1, par exemple) peuvent modifier la pente et/ou les coudes du plateau. Bien que le seuil inférieur de l'autorégulation cérébrale soit traditionnellement défini comme étant environ $50 \mathrm{mmHg}$, ce dernier est assez variable. Il peut en particulier être influencé par la présence d'une hypertension préexistante. ${ }^{2}$ Même en l'absence d'hypertension, l'hétérogénéité inter-patient au niveau de l'autorégulation (particulièrement au seuil inférieur), possiblement superposée à différents degrés de maladie vasculaire occlusive, remet en question la validité de définitions arbitraires d'une tension artérielle normale; dès lors, il est difficile de définir l'hypotension à l'aide d'un simple chiffre.

L'oxymétrie cérébrale, une technologie optique non effractive utilisant la NIRS, pourrait permettre de déterminer en temps réel les divers seuils d'autorégulation, ${ }^{3}$ ce qui pourrait avoir une pertinence dans la façon de définir une hypotension significative d'un point de vue clinique. À l'aide de principes semblables à ceux de l'oxymétrie de pouls conventionnelle (par ex. la loi de Beer-Lambert) qui permettent de déterminer les niveaux d'hémoglobine oxygénée et désoxygénée par l'absorption différentielle de diverses longueurs d'ondes dans l'infrarouge proche, ${ }^{4}$ l'oxymétrie cérébrale mesure la saturation en oxygène du sang cérébral régional (soit environ $75 \%$ de contenu veineux et $25 \%$ de contenu artériel) $\left(\mathrm{rSO}_{2}\right)$. Une diminution de $\mathrm{rSO}_{2}$ reflète un taux d'extraction d'oxygène élevé, qui peut être provoqué par un apport d'oxygène diminué (le coupable le plus commun) ou par une utilisation accrue de cet oxygène. Plusieurs variables physiologiques peuvent modifier la $\mathrm{rSO}_{2} \cdot{ }^{5}$ Par exemple, la réponse dynamique de la $\mathrm{rSO}_{2}$ aux changements de $\mathrm{PaCO}_{2}$ est bien connue, même lors d'une hypocapnie faible (c.-à-d. une $\mathrm{PaCO}_{2}<35 \mathrm{mmHg}$, ce qui est assez fréquent, et souvent ignoré, pendant des soins anesthésiques de routine) entraînant des réductions importantes du DSC et de l'apport d'oxygène ainsi que des réductions correspondantes de la $\mathrm{rSO}_{2}$.

L'oxymétrie cérébrale pourrait bientôt permettre d'optimiser la tension artérielle périopératoire, en fournissant des données qui permettraient de déterminer lorsque celle-ci est trop basse par exemple. Néanmoins, lorsqu'on pense aux implications de l'hypotension peropératoire, non seulement le seuil de tension (c.-à-d. la gravité de l'hypotension) doit-il être défini, mais également la durée passée en dessous de ce seuil; en intégrant ces concepts, on obtient la surface sous la courbe (SSC) comme indice global de l'exposition à l'hypotension. Ainsi, on aborde la question de savoir si une hypotension peropératoire brève mais significative (telle que celle qui peut être observée lors de l'induction de l'anesthésie par exemple) est aussi grave que des périodes d'hypotension prolongée mais peut-être moins prononcée (en termes de niveaux absolus de tension artérielle). Une fois de plus, il a été démontré qu'une mesure dérivée de l'oxymétrie cérébrale à NIRS reflétait l'impact de l'hypotension sur les devenirs. En effet, Fischer et coll., dans une étude chez des patients subissant une chirurgie de la crosse de l'aorte, ont démontré qu'il existait une relation significative entre les complications d'une part et la SSC et le temps sous la courbe pour plusieurs seuils de $\mathrm{rSO}_{2}$ d'autre part. ${ }^{6}$ En chirurgie non cardiaque, l'impact de périodes d'hypotension relativement légère bien que prolongée (souvent peu reconnue en raison de questions liées au positionnement du brassard de tension artérielle chez les patients en position assise) est désormais pris en compte grâce à l'oxymétrie cérébrale. Des données probantes obtenues de patients subissant une chirurgie orthopédique de l'épaule indiquent l'utilité de la NIRS pour dépister les épisodes d'hypotension moins grave mais potentiellement prolongée ainsi que leurs effets sur les devenirs. ${ }^{7}$

En se servant de la saturation en oxygène des tissus cérébraux comme mesure indirecte du DSC relatif, on pourrait déterminer la relation d'autorégulation entre la tension et le DSC. En effet, des données probantes associant une détérioration de l'autorégulation mesurée par la NIRS à des complications neurologiques après chirurgie cardiaque laissent supposer qu'un monitorage en temps réel a le potentiel de reconnaître les occasions de modifier le pronostic. ${ }^{8}$ Ainsi, la définition de la tension optimale de chaque personne pourrait remplacer l'approche plus généralisée d'une tension artérielle inhérente à toute la population dans son hétérogénéité. Malgré un corpus de littérature en croissance constante décrivant à répétitions une association entre une $\mathrm{rSO}_{2}$ détériorée et un pronostic périopératoire défavorable, ${ }^{6,9-12}$ les avantages potentiels d'une approche intuitive de personnalisation des soins, bien que très prometteuse, doivent encore être testés dans des études cliniques bien conçues et bien réalisées.

Tout comme il se peut qu'on doive repenser la définition et le traitement de l'hypotension et son contexte clinique, l'hypoxie, bien qu'étant à première vue une manifestation clinique évidente à définir, nécessite également qu'on s'y attarde du point de vue de l'oxymétrie cérébrale. L'hypoxémie, définie comme une pression partielle d'oxygène faible dans le sang, entraîne des niveaux d'oxygène tissulaire plus bas que la normale. Cependant, il existe clairement des conditions dans lesquelles le sang artériel contient suffisamment d'oxygène pour s'éloigner largement de la zone d'hypoxémie, mais dans lesquelles, au niveau tissulaire, on observe des niveaux d'oxygène insuffisants pour le métabolisme aérobie. Paradoxalement, certaines données probantes suggèrent également qu'une 
saturation en oxygène "normale » ne serait peut-être pas idéale pour tout le monde. Chez l'humain, on observe des tolérances variables à une hypoxie relative selon l'âge. Par exemple, chez les patients très jeunes, comme les nouveau-nés prématurés, il est possible d'observer une tolérance, bien que temporaire (c.-à-d. les premiers jours de vie) à un contenu d'oxygène plus bas, alors qu'un surplus d'oxygène pourrait nuire à ces mêmes patients. Les enfants atteints de cardiopathie congénitale cyanogène pourraient eux aussi souffrir d'une saturation en oxygène autrement normale.

Les informations concernant l'apport d'oxygène et son utilisation fournies par l'oxymétrie cérébrale nous donnent désormais d'autres pistes de réponses à la question de savoir si, en dépit d'une saturation artérielle systémique normale et d'une tension artérielle adaptée, un patient pourrait manifester une hypoxie tissulaire. Afin de garantir le maintien d'une saturation en oxygène optimale des tissus (et particulièrement du cerveau), il faut pouvoir évaluer en temps réel les nombreux facteurs déterminants de l'oxygénation tissulaire, notamment les facteurs liés à l'apport d'oxygène (c.-à-d. tension, débit sanguin et contenu en oxygène) et à son utilisation. Jusqu'à récemment, notre compréhension de la manière d'intégrer ces variables individuelles a été imparfaite et s'est fondée sur des suppositions générales. Grâce à l'oxymétrie cérébrale par spectroscopie proche infrarouge, on peut intégrer le contenu en oxygène (déterminé selon l'hémoglobine et la $\mathrm{PaO}_{2}$ ), le débit sanguin et la tension, ainsi que d'autres éléments liés au métabolisme tissulaire. Des soins personnalisés peuvent ainsi être offerts aux patients de plus en plus complexes qui se présentent pour recevoir des soins périopératoires.

Les cibles finales d'une définition de l'hypotension et de l'hypoxie pertinente (c.-à-d. l'utilité diagnostique de la NIRS) doivent être mises en contexte avec la façon dont les traitements s'orienteront (c.-à-d. l'utilité thérapeutique de la NIRS). Les interventions thérapeutiques sont inextricablement liées aux technologies diagnostiques, les unes étant guidées par les autres. Toutefois, l'enthousiasme provoqué par le potentiel de la NIRS pour améliorer le pronostic, est mitigé quelque peu par le fait qu'aucune stratégie de monitorage n'a jamais véritablement été liée à un meilleur devenir. En effet, un enthousiasme semblable par rapport à l'avantage généralisé du cathéter de Swan-Ganz, par exemple, a été brisé par les recherches menées au cours des décennies suivant son adoption. ${ }^{13}$ Même l'oxymétrie de pouls, une norme de soins dans notre pratique, n'a jamais été démontrée comme améliorant les devenirs, ${ }^{14}$ bien que cette modalité possède clairement un avantage intuitif. De plus, contrecarrant tout avantage potentiel de n'importe quel appareil de surveillance individuelle, il faut garder à l'esprit que c'est bien l'intervention thérapeutique (même si celle-ci est guidée par l'appareil) qui pourrait entraîner un changement du devenir, et non l'appareil en tant que tel.

La complexité en constante évolution du monitorage physiologique périopératoire (et les options thérapeutiques associées), apparue à la lumière des nouvelles technologies s'appuyant sur la NIRS, indique que notre compréhension auparavant statique de l'hypotension et de l'hypoxie a clairement besoin d'être repensée. L'oxymétrie cérébrale est un bon exemple de ces enjeux et sera sans aucun doute au centre de débats quant à savoir si l'évolution du patient pourra être optimisée et comment. Ceci étant dit, il semble que le vieil adage en anesthésie, d'éviter l'hypotension et l'hypoxie, soit à nouveau suffisamment pertinent et que nous devions garder l'esprit ouvert dans notre approche de cette question.

Competing interests None declared.

\section{References}

1. Newman MF, Croughwell ND, White WD, et al. Effect of perfusion pressure on cerebral blood flow during normothermic cardiopulmonary bypass. Circulation 1996; 94: II353-7.

2. Strandgaard S. Autoregulation of cerebral blood flow in hypertensive patients. The modifying influence of prolonged antihypertensive treatment on the tolerance to acute, druginduced hypotension. Circulation 1976; 53: 720-7.

3. Brady KM, Mytar JO, Lee JK, et al. Monitoring cerebral blood flow pressure autoregulation in pediatric patients during cardiac surgery. Stroke 2010; 41: 1957-62.

4. Jobsis FF. Noninvasive, infrared monitoring of cerebral and myocardial oxygen sufficiency and circulatory parameters. Science 1977; 198: 1264-7.

5. Denault A, Deschamps A, Murkin JM. A proposed algorithm for the intraoperative use of cerebral near-infrared spectroscopy. Semin Cardiothorac Vasc Anesth 2007; 11: 274-81.

6. Fischer GW, Lin HM, Krol M, et al. Noninvasive cerebral oxygenation may predict outcome in patients undergoing aortic arch surgery. J Thorac Cardiovasc Surg 2011; 141: 815-21.

7. Murphy GS, Szokol JW, Marymont JH, et al. Cerebral oxygen desaturation events assessed by near-infrared spectroscopy during shoulder arthroscopy in the beach chair and lateral decubitus positions. Anesth Analg 2010; 111: 496-505.

8. Joshi B, Brady K, Lee J, et al. Impaired autoregulation of cerebral blood flow during rewarming from hypothermic cardiopulmonary bypass and its potential association with stroke. Anesth Analg 2010; 110: 321-8.

9. Casati A, Fanelli G, Pietropaoli P, et al. Continuous monitoring of cerebral oxygen saturation in elderly patients undergoing major abdominal surgery minimizes brain exposure to potential hypoxia. Anesth Analg 2005; 101: 740-7.

10. Heringlake $M$, Garbers $C$, Kabler JH, et al. Preoperative cerebral oxygen saturation and clinical outcomes in cardiac surgery. Anesthesiology 2011; 114: 58-69.

11. Slater JP, Guarino T, Stack J, et al. Cerebral oxygen desaturation predicts cognitive decline and longer hospital stay after cardiac surgery. Ann Thorac Surg 2009; 87: 36-44. 
12. Murkin JM, Adams SJ, Novick RJ, et al. Monitoring brain oxygen saturation during coronary bypass surgery: a randomized, prospective study. Anesth Analg 2007; 104: 51-8.

13. Sandham JD, Hull RD, Brant RF, et al. A randomized, controlled trial of the use of pulmonary-artery catheters in high-risk surgical patients. N Engl J Med 2003; 348: 5-14.
14. Moller JT, Pedersen T, Rasmussen LS, et al. Randomized evaluation of pulse oximetry in 20, 802 patients: I. Design, demography, pulse oximetry failure rate, and overall complication rate. Anesthesiology 1993; 78: 436-44. 